surstaos

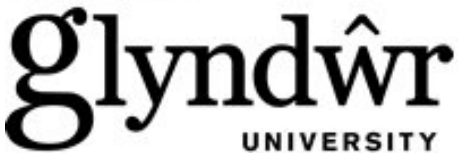

Glyndŵr University

Glyndŵr University Research Online

Computing

Computer Science

$4-1-2007$

\title{
CoLoRaDe: A Novel Algorithm for Controlling Long-Range Dependent Network Traffic
}

Karim Mohammed Rezaul

Vic Grout

Glyndwr University, v.grout@glyndwr.ac.uk

Follow this and additional works at: http://epubs.glyndwrac.uk/cair

Part of the Computer and Systems Architecture Commons, Digital Communications and Networking Commons, Hardware Systems Commons, and the Systems and Communications Commons

\section{Recommended Citation}

Rezaul, K.M. \& Grout, V. (2007), 'CoLoRaDe: A Novel Algorithm for Controlling Long-Range Dependent Network Traffic'. [Paper presented to the Sixth International Conference on Networking (ICN 2007) 22nd -28th April 2007]. Plymouth: Plymouth University

This Conference Paper is brought to you for free and open access by the Computer Science at Glyndŵr University Research Online. It has been accepted for inclusion in Computing by an authorized administrator of Glyndŵr University Research Online. For more information, please contact d.jepson@glyndwr.ac.uk. 


\title{
CoLoRaDe: A Novel Algorithm for Controlling Long-Range Dependent Network Traffic
}

\begin{abstract}
Long-range dependence characteristics have been observed in many natural or physical phenomena. In particular, a significant impact on data network performance has been shown in several papers. Congested Internet situations, where TCP/IP buffers start to fill, show long-range dependent (LRD) self-similar chaotic behaviour. The exponential growth of the number of servers, as well as the number of users, causes the performance of the Internet to be problematic since the LRD traffic has a significant impact on the buffer requirements. The Internet is a large-scale, wide-area network for which the importance of measurement and analysis of traffic is vital. The intensity of the long-range dependence (LRD) of communications network traffic can be measured using the Hurst parameter. A variety of techniques (such as R/S analysis, aggregated variance-time analysis, periodogram analysis, Whittle estimator, Higuchi's method, wavelet-based estimator, absolute moment method, etc.) exist for estimating Hurst exponent but the accuracy of the estimation is still a complicated and controversial issue. Earlier research (Rezaul et al., 2006) introduced a novel estimator called the Hurst exponent from the autocorrelation function (HEAF) and it was shown why lag 2 in HEAF (i.e. HEAF (2)) is considered when estimating LRD of network traffic. HEAF estimates $\mathrm{H}$ by a process which is simple, quick and reliable. In this research we extend these concepts by introducing a novel algorithm for controlling the long-range dependence of network traffic, named CoLoRaDe which is shown to reduce the LRD of packet sequences at the router buffer.
\end{abstract}

\section{Keywords}

Long-Range Dependent Network Traffic, traffic shaping, self-similar network traffic

\author{
Disciplines \\ Computer and Systems Architecture | Digital Communications and Networking | Hardware Systems | \\ Systems and Communications
}

\section{Comments}

Copyright (C) 2007 IEEE - All Rights reserved. This paper was presented to Sixth International Conference on Networking (ICN 2007) 22nd -28th April 2007 in Martinique, French Caribbean. The proceedings were published by the IEEE and are available at http://ieeexplore.ieee.org This material is posted here with permission of the IEEE and the author. Such permission of the IEEE does not in any way imply IEEE endorsement of any of the products or services of Glyndwr University Wrexham. Internal or personal use of this material is permitted. However, permission to reprint/republish this material for advertising or promotional purposes or for creating new collective works for resale or redistribution must be obtained from the IEEE by writing to pubs-permissions@ieee.org. By choosing to view this document, you agree to all provisions of the copyright laws protecting it. 


\section{CoLoRaDe: A Novel Algorithm for Controlling Long- range Dependent Network Traffic}

\author{
Karim Mohammed Rezaul \\ Centre for Applied Internet Research \\ (CAIR) \\ University of Wales, NEWI \\ Plas Coch Campus, Wrexham, UK \\ morekba786@yahoo.co.uk
}

\author{
Vic Grout \\ Centre for Applied Internet Research \\ (CAIR) \\ University of Wales, NEWI \\ Plas Coch Campus, Wrexham, UK \\ v.grout@newi.ac.uk
}

\begin{abstract}
Long-range dependence characteristics have been observed in many natural or physical phenomena. In particular, a significant impact on data network performance has been shown in several papers. Congested Internet situations, where TCP/IP buffers start to fill, show long-range dependent (LRD) self-similar chaotic behaviour. The exponential growth of the number of servers, as well as the number of users, causes the performance of the Internet to be problematic since the LRD traffic has a significant impact on the buffer requirements. The Internet is a large-scale, widearea network for which the importance of measurement and analysis of traffic is vital. The intensity of the long-range dependence (LRD) of communications network traffic can be measured using the Hurst parameter. A variety of techniques (such as $\mathbf{R} / \mathbf{S}$ analysis, aggregated variance-time analysis, periodogram analysis, Whittle estimator, Higuchi's method, Wavelet-based estimator, absolute moment method, etc.) exist for estimating Hurst exponent but the accuracy of the estimation is still a complicated and controversial issue. Earlier research [1] introduced a novel estimator called the Hurst Exponent from the Autocorrelation Function (HEAF) and it was shown why lag 2 in HEAF (i.e. HEAF (2)) is considered when estimating LRD of network traffic. HEAF estimates $H$ by a process which is simple, quick and reliable. In this research we extend these concepts by introducing a novel algorithm for controlling the long-range dependence of network traffic, named CoLoRaDe which is shown to reduce the LRD of packet sequences at the router buffer.
\end{abstract}

\section{INTRODUCTION}

The importance of Long-Range Dependence ( $L R D)$ in traffic engineering problems, such as traffic measurement, queuing behaviour and buffer sizing, admission control and congestion control, is vital. The research in [2] shows that the consequences of LRD are packet delays and application level delays that cause a heavy-tailed distribution. TCP estimates the round trip timer values from the peer acknowledgements and as a result congestions appear more frequently while maintaining the impulsive behaviour with increase in load. The influence of LRD properties on the delay performance at packet and application level is reported in [3] and metrics of network performance, such as throughput, packet loss, latency and buffer occupancy levels, are affected by the presence of LRD phenomenon across many types of networks. The work in [3] also claims that packet delay behaviour tends to be more heavy-tailed in the case of LRD traffic while the congestion window size is increased. The impact of LRD on quality of service (QoS) has been analysed in [4] showing that, the greater the LRD, the lower the QoS.
The LRD property of traffic fluctuations has important implications on the performance, design and dimensioning of the network [5]. A simple, direct parameter, characterizing the degree of long-range dependence, is the Hurst parameter. The Hurst exponent (or Hurst parameter, $H$ ), which more than a half-century ago was proposed for analysis of long-term storage capacity of reservoirs [6], is used today to measure the intensity of LRD in network traffic. A number of methods have been proposed to estimate the Hurst parameter. Some of the most popular include the aggregated variance time (V/T) [7], Rescaledrange (R/S) [5, 6], Higuchi method [8], wavelet-based method $[9,10]$ although there are many others. In all these methods, $H$ is calculated by taking the slope from a log-log plot. Over time, the wavelet-based Hurst parameter has acquired popularity in estimating LRD traffic. However the study [11] explored the advantages and limitations of wavelet estimators and found that a traffic trace with a number of deterministic shifts in the mean rate results in a steep wavelet spectrum, which leads to an overestimate of the Hurst parameter. The intensity of long-range dependence is measured fr file size or document size [12], packet counts (number of packets per unit time) [13, 14, 15], interarrival time [16, 17], frame size [18], connection size [19], packet length [20], number of bytes per unit time [5], Bit or byte rate [21] amongst others..

This paper is organised as follows. Section II describes the definitions of self-similarity, long-range dependence and the autocorrelation function. Section III elaborates the HEAF estimator. Section IV introduces the algorithm CoLoRaDe and its function. Section $\mathrm{V}$ depicts the complexity of the CoLoRaDe by experimental analysis. Finally we draw a conclusion and suggest future works in section VI.

\section{SELF-SIMILARITY, LONG-RANGE DEPENDENCE AND AUTOCORRELATION FUNCTION}

In general two or more objects having the same characteristics are called self-similarity. A phenomenon that is self-similar looks the same or behaves the same when viewed at different degrees of magnification or different scales on a dimension and bursty over all time scales. Selfsimilarity is the property of a series of data points to retain a pattern or appearance regardless of the level of granularity used and is the result of long-range dependence in the data series. If a self-similar process is bursty at a wide range of timescales, it may exhibit long-range- dependence. In general lagged autocorrelations are used in time series 
analysis for empirical stationary tests. Self-similarity manifests itself as long-range dependence (i.e., long memory) in the time series of arrivals. The evidence of very slow, linear decay in the sample lag autocorrelation finction (ACF) indicates the nonstationary behaviour [22]. The research [23] show that Internet traffic is nonstationary.

Long-range-dependence means that all the values at any time are correlated in a positive and non-negligible way with values at all future instants. For a continuous time process $Y=\{Y(t), t \geq 0\}$ is self-similar if it satisfies the following condition [24]:

$$
Y(t) \stackrel{d}{=} a^{-H} Y(a t), \quad \forall a>0, \text { and } 0<H<1
$$

where $H$ is the index of self-similarity, called Hurst parameter and the equality is in the sense of finitedimensional distributions.

The stationary process $X$ is said to be a long-range dependent process if its autocorrelation function (ACF) is non-summable [25] meaning that $\sum_{k=-\infty}^{\infty} \rho_{k}=\infty$

The details of how ACF decays with $k$ are of interest because the behaviour of the tail of ACF completely determines its summability. According to [5], $X$ is said to exhibit long-range dependence if

$\rho_{k} \sim L(t) k^{-(2-2 H)}$, as $k \rightarrow \infty$

where $\frac{1}{2}<H<1$ and $L($.) slowly varies at infinity, i.e.,

$$
\lim _{t \rightarrow \infty} \frac{L(x t)}{L(t)}=1, \quad \text { for all } x>0
$$

Equation (2.1) implies that the LRD is characterized by an autocorrelation function that decays hyperbolically rather than exponentially fast.

LRD processes are characterized by a slowly decaying covariance function that is no more summable. When the network performance is affected by LRD the data are correlated over an unlimited range of time lags and this property results in a scale invariance phenomenon. Then no characteristic time scale can be identified in the process, they are all equivalent for describing its statistics, i.e., the part resembles the whole and vice e versa. This is why lrd is also called Self-Similarity [26].

\section{HEAF: A 'HURST EXPONENT BY AUTOCORRELATION FUNCTION' ESTIMATOR}

A new estimator has been introduced [1] by extending the approach of Kettani and Gubner [27]. As in [27], for a given observed data $X_{i}$ (i.e. $X_{1}, \ldots \ldots \ldots, X_{n}$ ), the sample autocorrelation function can be calculated by the following method:

$$
\begin{aligned}
& \text { Let } \hat{\mu}_{n}=\frac{1}{n} \sum_{i=1}^{n} X_{i} \\
& \text { and } \hat{\gamma}_{n}(k)=\frac{1}{n} \sum_{i=1}^{n-k}\left(x_{i}-\hat{\mu}_{n}\right)\left(x_{i+k}-\hat{\mu}_{n}\right),
\end{aligned}
$$

where $k=0,1,2, \ldots ., n$,

with $\hat{\sigma}_{n}^{2}=\hat{\gamma}_{n}(0)$.
Then the sample autocorrelations of lag $k$ are given by

$$
\hat{\rho}_{k}=\frac{\hat{\gamma}_{n}(k)}{\hat{\sigma}_{n}^{2}}
$$

(Equations (3.1), (3.2), (3.3) and (3.4) denote the sample mean, the sample covariance, the sample variance and the sample autocorrelation, respectively). A second-order stationary process is said to be exactly second-order selfsimilar with Hurst exponent $1 / 2<H<1$ if

$\rho_{k}=0.5\left[(k+1)^{2 H}-2 k^{2 H}+(k-1)^{2 H}\right]$

From equation (3.5), Kettani and Gubner suggest a moment estimator of $H$. They consider the case where $k=1$ and replace $\rho_{1}$ by its sample estimate $\hat{\rho}_{1}$, as defined in equation (3.4). This gives an estimate for $H$ of the form

$\hat{H}=\frac{1}{2}+\frac{1}{2 \log _{e} 2} \log e^{\left(1+\hat{\rho}_{1}\right)}$

Clearly, this estimate is straightforward to evaluate, requiring no iterative calculations. For more details of the properties of this estimator, see Kettani and Gubner [27].

An alternative estimator of $H$ is proposed based upon equation (3.5), by considering the cases where $k>1$. Note that the sample equivalent of equation (3.5) can be expressed as

$$
\begin{aligned}
f(H)= & \hat{\rho}_{k}-0.5\left\{(k+1)^{2 H}\right. \\
& \left.-2 k^{2 H}+(k-1)^{2 H}\right\}=0 .
\end{aligned}
$$

Thus, for a given observed $\hat{\rho}_{k}, k>1$, a suitable numerical procedure can be used to solve this equation, and find an estimate of $H$. This is denoted as a $\operatorname{HEAF}(k)$ estimate of $H$.

To solve equation (3.7) for $H$ the well-known NewtonRaphson (N-R) method is used. This requires the derivative of $f(H)$. Here note that $k$ ? ,

$$
f^{\prime}(H)=-0.5\left\{\begin{array}{c}
(2 \log (k+1))(k+1)^{2 H} \\
-(4 \log (k))(k)^{2 H}+ \\
(2 \log (k-1))(k-1)^{2 H}
\end{array}\right\}
$$

Hence, the algorithm to estimate $\operatorname{HEAF}(\mathrm{k})$, for any lag $k$, consists of the following steps:

1. Compute the sample autocorrelations for lag $k$ of a given data set by equation (3.4). (Note that $X_{i}$ can be denoted as the number of bits, bytes, packets or bit rates observed during the $i$ th interval. If $X_{i}$ is a Gaussian process, it is known as fractional Gaussian noise).

2. Make an initial guess of $H$, e.g. $H_{l}=0.6$, then calculate $H_{2}, \quad H_{3}, \quad H_{4, \ldots . .}, \quad$ successively using $H_{r+1}=H_{r}-f\left(H_{r}\right) / f^{\prime}\left(H_{r}\right)$, until convergence, to find the estimate $\hat{H}$ for the given lag $k$. An initial consideration is of the case where $k=2$ in equation (3.2); i.e. $\operatorname{HEAF}(2)$ is considered first.

One of the major advantages of the HEAF estimator is speed, as the NR-method converges very quickly to a root. There is no general convergence criterion for NR. Its convergence depends on the nature of the function and on the accuracy of the initial guess. Fortunately the form of the function (i.e., equation (3.7)) appears to converge quickly (within at most four iterations) for any initial guess in the 
range of interest, namely $\mathrm{H}$ in $(0.2,1)$. If an iteration value, $\mathrm{Hr}$ is such that $f^{\prime}\left(H_{r}\right) \cong 0$, then one can face "division by zero" or a near-zero number. This will give a large magnitude for the next value, $H_{r+1}$ which in turn stops the iteration. This problem can be resolved by increasing the tolerance parameter in the $\mathrm{N}-\mathrm{R}$ program. $\mathrm{A} \operatorname{HEAF}(\mathrm{k})$, for $\mathrm{k}$ $=2, \ldots, 11$, have been considered and no difficulty in finding the root in $(0.5,1)$ have been encountered.

\section{COLORADE: AN ALGORITHM FOR CONTROLLING LRD TRAFFIC}

Figure 1 illustrates a schematic view of the operation of the CoLoRaDe algorithm at the router buffer.

Here $\mathrm{P}_{1}, \mathrm{P}_{2}, \mathrm{P}_{3}, \ldots \ldots, \mathrm{P}_{\mathrm{n}}$ are the slots of the packet sequences.

$\mathrm{S}_{1}, \mathrm{~S}_{2}, \mathrm{~S}_{3}, \ldots \ldots \ldots, \mathrm{S}_{\mathrm{n}}$ are the sets constructed by shuffling the slots of the packet sequences.

$\mathrm{P}_{1} \mathrm{~S}, \mathrm{P}_{2} \mathrm{~S}, \mathrm{P}_{3} \mathrm{~S}, \ldots \ldots, \mathrm{P}_{\mathrm{n}} \mathrm{S}$ are the blocks (groups) of the sets of the slots $\mathrm{P}_{1}, \mathrm{P}_{2}, \mathrm{P}_{3}, \ldots \ldots, \mathrm{P}_{\mathrm{n}}$ respectively.

$\left(\mathrm{P}_{1} \mathrm{~S}\right)_{\operatorname{minH}}, \quad\left(\mathrm{P}_{2} \mathrm{~S}\right)_{\operatorname{minH}}, \quad\left(\mathrm{P}_{3} \mathrm{~S}\right)_{\operatorname{minH}}, \ldots \ldots .,\left(\mathrm{P}_{\mathrm{n}} \mathrm{S}\right)_{\operatorname{minH}}$ are the individual sets of packet sequences from the blocks (i.e. $\mathrm{P}_{1} \mathrm{~S}, \mathrm{P}_{2} \mathrm{~S}, \mathrm{P}_{3} \mathrm{~S}, \ldots \ldots, \mathrm{P}_{\mathrm{n}} \mathrm{S}$ ) which possess the minimum Hurst parameter. In other words, each block (e.g. $\mathrm{P}_{1} \mathrm{~S}$ ) consists of several sets where one of the sets possesses the minimum value of Hurst parameter.

Let us assume that the client networks (such as $C_{1}, C_{2}$, $\mathrm{C}_{3}, \ldots, \mathrm{C}_{\mathrm{n}}$ ) are connected to the main Internet service provider (ISP) router. The packet sequences from different sources are queued at the point $Q$. Then the packet sequences are slotted into various length (e.g. $\mathrm{N}=12, \mathrm{~N}=$ $25, \mathrm{~N}=50$ etc.) sequences. Each slot of these sequences is shuffled for a particular number of times so that it has several sets. Then the Hurst parameter $(\mathrm{H})$ for each set of a slot is estimated. In other words, H's have been estimated for $\mathrm{P}_{1} \mathrm{~S}$ (i.e. $\mathrm{P}_{1} \mathrm{~S}_{1}, \mathrm{P}_{1} \mathrm{~S}_{2}, \mathrm{P}_{1} \mathrm{~S}_{3}, \ldots \ldots, \mathrm{P}_{1} \mathrm{~S}_{\mathrm{n}}$ ), $\mathrm{P}_{2} \mathrm{~S}, \mathrm{P}_{3} \mathrm{~S}, \ldots \ldots \ldots$, $\mathrm{P}_{\mathrm{n}} \mathrm{S}$ respectively and will be scheduled to the transmitter according to $\left(\mathrm{P}_{1} \mathrm{~S}\right)_{\operatorname{minH}},\left(\mathrm{P}_{2} \mathrm{~S}\right)_{\operatorname{minH}},\left(\mathrm{P}_{3} \mathrm{~S}\right)_{\operatorname{minH}}, \ldots \ldots,\left(\mathrm{P}_{\mathrm{n}} \mathrm{S}\right)_{\operatorname{minH}}$ and finally sent out to the core network (i.e. Internet) on a FIFO basis as shown in figure. The CoLoRaDe algorithm is given in Table I.

The algorithm is implemented in Java and a sample output given in Table II. Here the impact of Hurst estimates on the queuing process can be observed

\section{COMPLEXITY OF THE ALGORITHM, COLORADE}

To explore the complexity of CoLoRaDe, we chose six workstations with different specifications which are represented in Table III. We investigated several lengths of packet sequences such as $\mathrm{N}=1000, \mathrm{~N}=2000, \mathrm{~N}=3000, \mathrm{~N}$ $=5000, \mathrm{~N}=10000, \mathrm{~N}=15000, \mathrm{~N}=20000, \mathrm{~N}=25000, \mathrm{~N}=$ $30000, \mathrm{~N}=35000, \mathrm{~N}=40000, \mathrm{~N}=45000$ and $\mathrm{N}=50000$. According to CoLoRaDe, these length sequences have been slotted by considering a certain number of samples (NS). For instance, for $\mathrm{N}=1000$, we slot this length of sequences by $\mathrm{NS}=12, \mathrm{NS}=25, \mathrm{NS}=50, \mathrm{NS}=100, \mathrm{NS}=200, \mathrm{NS}=500$ and NS $=1000$. Similar procedures have been followed for other types of length of sequences. In our research we mainly concentrate on the time complexity of the algorithm.
A router introduces delay (latency) as it processes the packets it receives. Consequently, time is a crucial factor here as we cannot not accept increased delay in processing the packets. Figure 2 represents the elapsed time observed using different PC's for a particular length of packet sequences where we consider different number of samples (NS) in each slot. It is clear that smaller numbers of samples per slot in the length of packet sequences contribute to longer periods of elapsed time to execute the algorithm. NS $=200$ per slot gives the best performance as the algorithm takes the least time to execute in this case when using PC2, PC3, PC4, PC5 and PC6.

Table I. The ColoRaDe algorithm

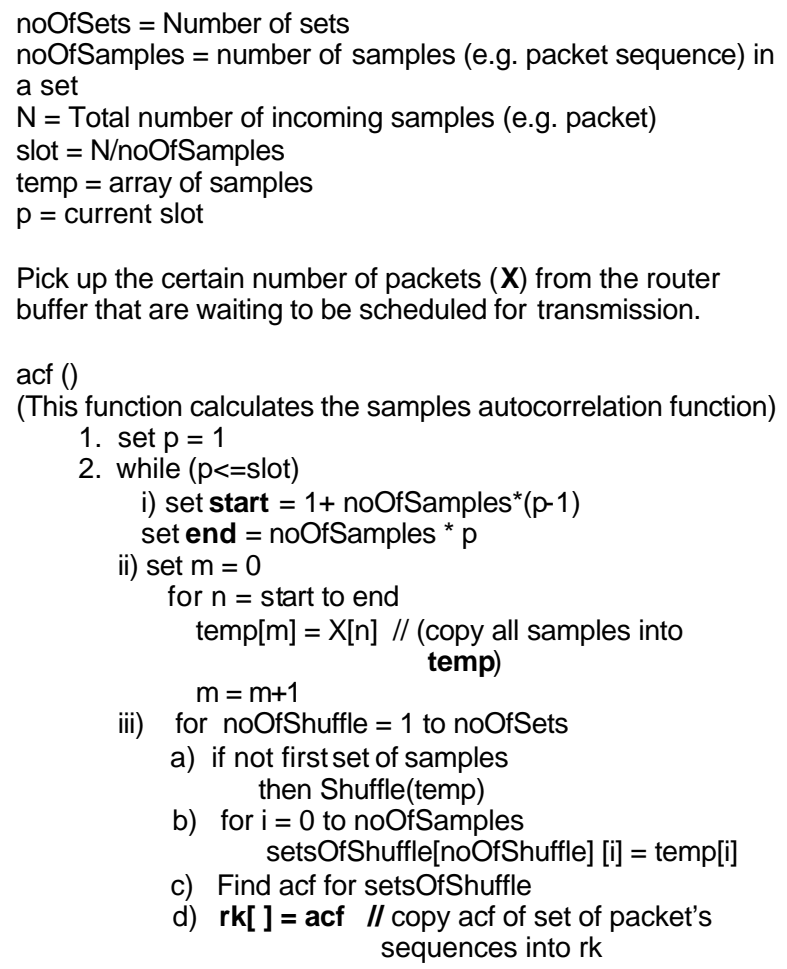

Pick up the certain number of packets $(\mathbf{X})$ from the router buffer that are waiting to be scheduled for transmission.

acf ()

(This function calculates the samples autocorrelation function)

1. set $p=1$

2. while $(p<=$ slot $)$

i) set start $=1+$ noOfSamples ${ }^{*}(p-1)$

set end $=$ noOfSamples ${ }^{*} p$

ii) set $m=0$

for $\mathrm{n}=$ start to end

temp $[\mathrm{m}]=X[\mathrm{n}] / /$ (copy all samples into

$\mathrm{m}=\mathrm{m}+1$

temp)

iii) for noOfShuffle $=1$ to noOfSets

a) if not first set of samples then Shuffle(temp)

b) for $\mathrm{i}=0$ to noOfSamples setsOfShuffle[noOfShuffle] [i] $=$ temp[i]

c) Find acf for setsOfShuffle

d) $\mathbf{r k}[$ ] = acf // copy acf of set of packet's sequences into rk

e) call Heaf (rk[], Hursts[], noOfHurstParameter)

iv) Find out the minimum Hurst parameter from the Hursts of all sets of samples (e.g. packet sequence)

v) Find the set that corresponds to minimum Hurst parameter

vi) Transmit the set (of the packets) that contains minimum Hurst

vii) $p=p+1$ (increment of slot number)

3. Go to step 1 until the packets awaiting at the router buffer

4. End of acf ()

Heaf (rk], Hursts[],noOfHurstParameter): this function estimates the Hurst parameter by $\operatorname{HEAF}(2)$ method for a given samples.

Shuffle (array): this function shuffles the set of samples main (): this is the main method which calls acf () 


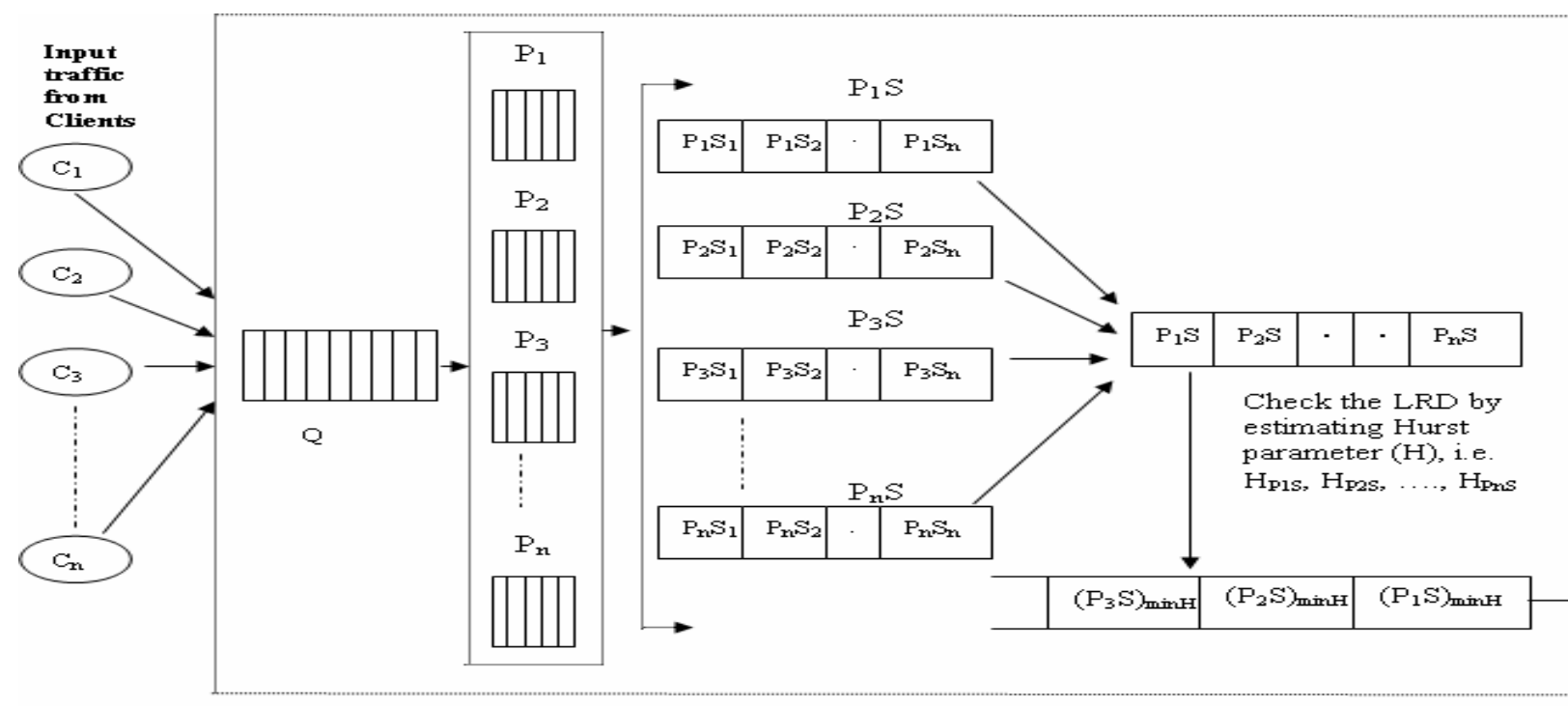

Main Router

Fig. 1. Illustration of packets management for controlling LRD at router buffer

Table II. Sample output by CoLoRaDe algorithm

\begin{tabular}{|c|c|c|c|c|c|}
\hline Set \# 1 & Set \# 2 & Set \# .3 & Set \#4 & Set \# 5 & Set \# 6 \\
\hline 40.0 & 40.0 & 72.0 & 72.0 & 552.0 & 40.0 \\
\hline 45.0 & 80.0 & 40.0 & 72.0 & 72.0 & 80.0 \\
\hline 552.0 & 44.0 & 40.0 & 40.0 & 44.0 & 41.0 \\
\hline 40.0 & 72.0 & 552.0 & 40.0 & 80.0 & 72.0 \\
\hline 72.0 & 40.0 & 72.0 & 41.0 & 40.0 & 40.0 \\
\hline 80.0 & 72.0 & 80.0 & 44.0 & 80.0 & 552.0 \\
\hline 41.0 & 45.0 & 40.0 & 552.0 & 41.0 & 40.0 \\
\hline 40.0 & 552.0 & 44.0 & 80.0 & 40.0 & 44.0 \\
\hline 72.0 & 40.0 & 41.0 & 40.0 & 40.0 & 45.0 \\
\hline 44.0 & 80.0 & 45.0 & 40.0 & 45.0 & 40.0 \\
\hline 80.0 & 40.0 & 40.0 & 80.0 & 72.0 & 80.0 \\
\hline 40.0 & 41.0 & 80.0 & 45.0 & 40.0 & 72.0 \\
\hline $\begin{array}{c}\text { Estima } \\
0.665 \\
0.6950 \\
0.6718 \\
0.6465 \\
0.6886 \\
0.6736\end{array}$ & $\begin{array}{l}\text { Hurst pa } \\
7669965 \\
3356916 \\
5470954 \\
0923490 \\
5520389 \\
3256908\end{array}$ & $\begin{array}{l}\text { meter for } \\
9 \\
3 \\
8 \\
9 \\
1\end{array}$ & sets are & & \\
\hline \multicolumn{6}{|c|}{ *** Minimum Hurst is 0.6465780923490018} \\
\hline $\begin{array}{l}\text { The co } \\
72.0 \\
72.0 \\
40.0 \\
40.0 \\
41.0 \\
44.0 \\
552.0 \\
80.0 \\
40.0 \\
40.0 \\
80.0 \\
45.0\end{array}$ & ponding & t (ready & cansmit) & & \\
\hline
\end{tabular}

Figure 3 depicts the elapsed time for adifferent length of packet sequences while the performance is observed with different PC's. Number of packet sequences (NS) in each slot considered here are $\mathrm{NS}=50, \mathrm{NS}=100, \mathrm{NS}=200$ and
$\mathrm{NS}=500$. It is clear that PC5 outperforms for all cases as it contains higher specifications.

Table III. Workstations with different specification

\begin{tabular}{|l|l|}
\hline Work station & \multicolumn{1}{|c|}{ Specification } \\
\hline pc1 & Intel Pentium (R) 4, CPU 2.4 GHz, 512 MB of RAM \\
\hline pc2 & Intel Pentium (R) 4, CPU 3.0 GHz, 0.99 GB of RAM \\
\hline pc3 & Intel Pentium (R) 4, CPU 3.0 GHz, 504 MB of RAM \\
\hline pc4 & Intel Pentium (R) 3, CPU 866 MHz, 384 MB of RAM \\
\hline pc5 & $\begin{array}{l}\text { Intel Centrino Duo Core, CPU T2250 @ 1.73 GHz, } \\
1024 \text { MB of RAM }\end{array}$ \\
\hline pc6 & Intel Pentium (R) 4, CPU 1.80 GHz, 256 MB of RAM \\
\hline
\end{tabular}

\section{CONCLUSIONS AND FUTURE WORK}

In this research we introduce a novel algorithm called CoLoRaDe to control the intensity of LRD traffic. Experimental results show that the CoLoRaDe is capable of reducing the LRD of packet sequences received at the router buffer before they are transmitted to the core network (i.e. Internet). The complexity analyses of CoLoRaDe suggest that the number of packet sequences (NS) in each set of a slot should be around NS $=200$ which makes the best value to execute the algorithm faster. To estimate the Hurst parameter, we used the process of HEAF (2) estimator, which is simple, reliable and capable of yielding quick estimation. I potentially can be used for real-time traffic measurement and control at the edge routers. As the main function of the CoLoRaDe algorithm is to reduce the LRD of packet traffic, it can contribute in reducing the network load towards the improvement of quality of service of future Internet. Future work will include evaluation of the applicability of the CoLoRaDe algorithm for real-time implementations in routers. 

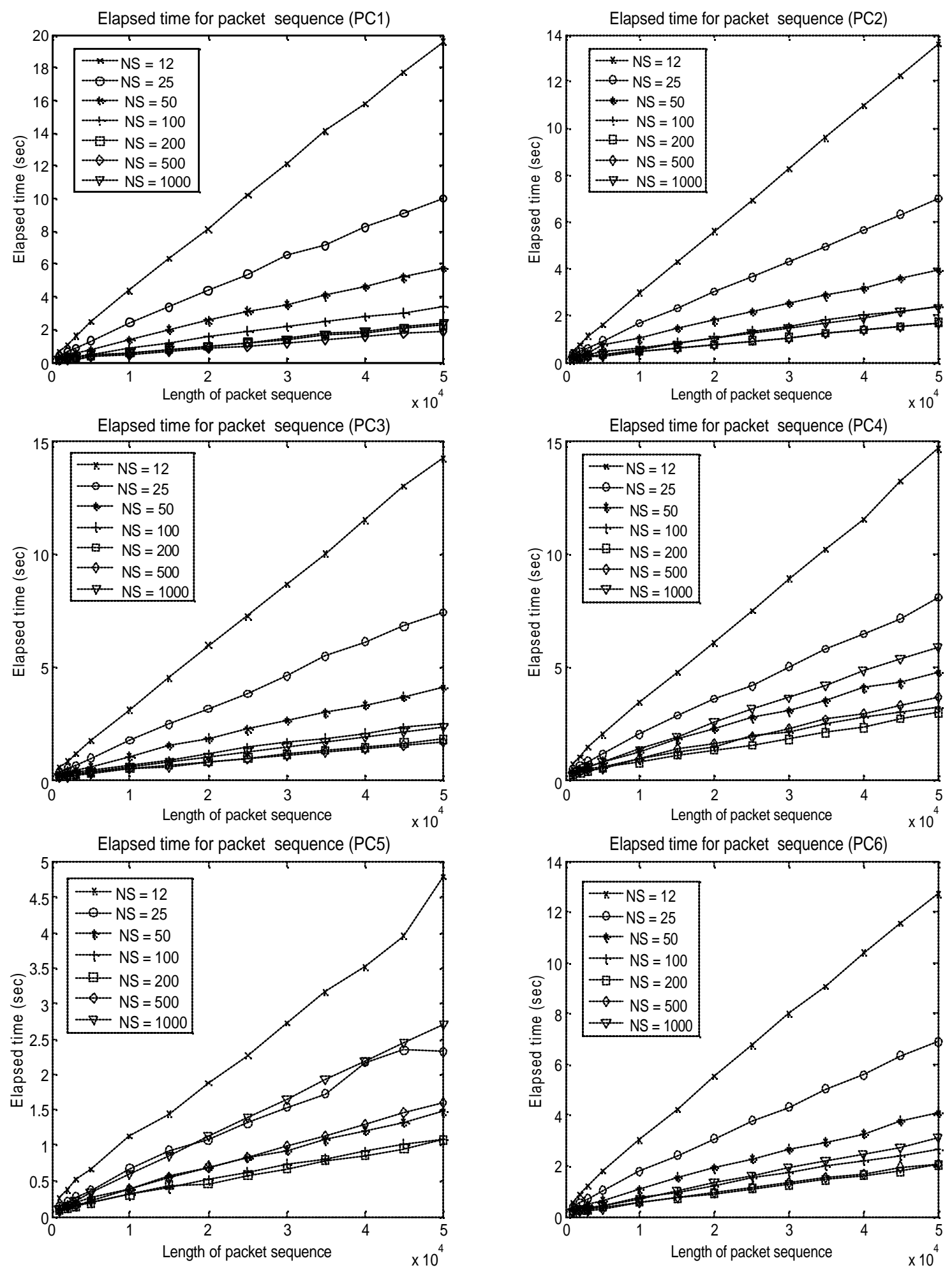

Fig. 2. Elapsed time for different length of packet sequences where each block or slot contains different length of sequences (e.g. NS $=12$ indicates 12 different packet sequences in each slot.) 

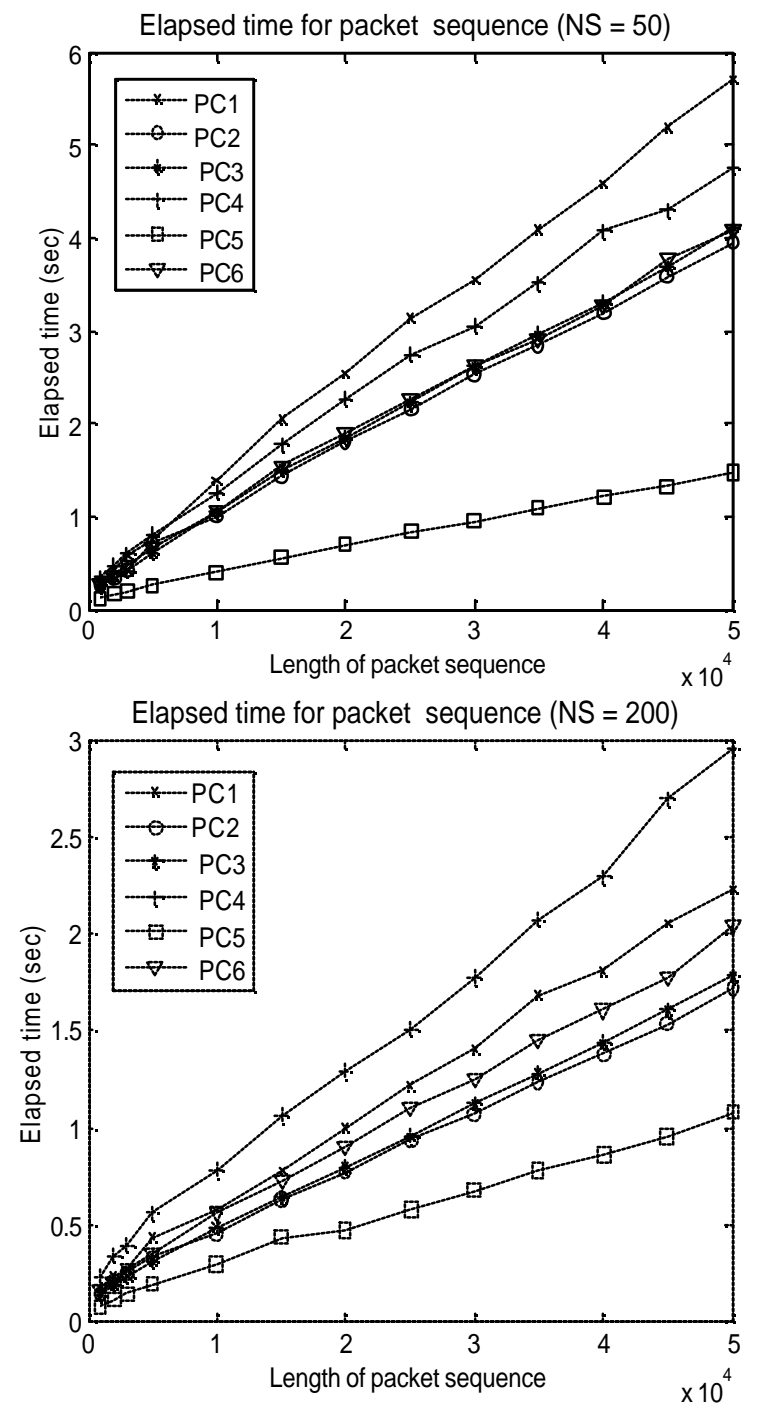
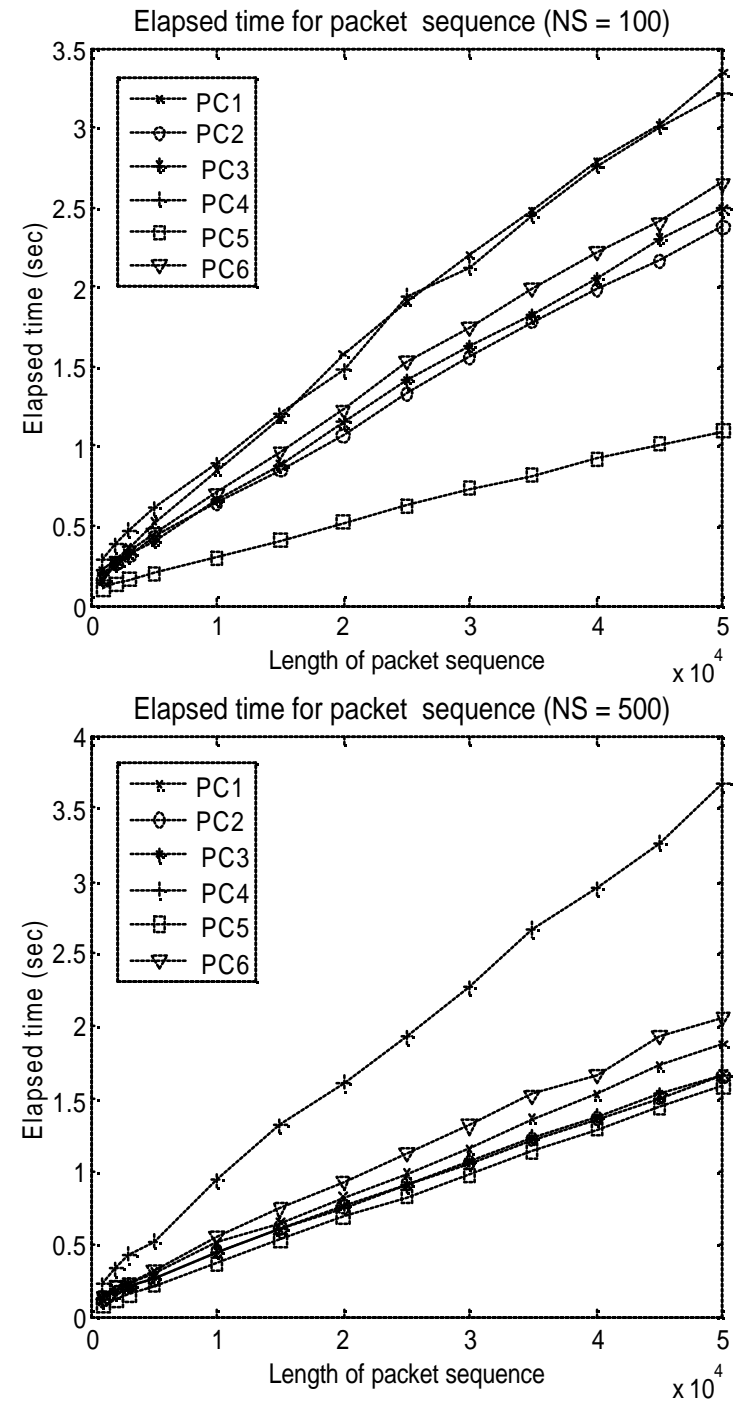

Fig. 3. Elapsed time for different length of packet sequences while performance is observed with different PC's.

\section{REFERENCES}

[1] Karim M. Rezaul, Algirdas Pakštas, Robert Gilchrist, Thomas M. Chen, HEAF: A Novel Estimator for Long-Range Dependent Selfsimilar Network Traffic, Y. Koucheryavy, J. Harju, and V.B. Iversen (Eds.): Next Generation Teletraffic and Wired/Wireless Advanced Networking (NEW2AN), May 29 - June 2, 2006, LNCS 4003, pp. 34- 45.

[5] Leland Will E. Taqqu M. S., Willinger W. and Wilson D. V., On the Self-similar nature of Ethernet Traffic (Extended version), IEEE/ACM Transactions on Networking, February 1994, Vol. 2, No. 1, pp. 1-15.

[2] Willinger, W., Paxson, V. and Taqqu, M.S., Self-Similarity and Heavy-tails: Struct ural Modeling of Network Traffic, A Practical Guide to Heavy Tails, 1998, Birkhauser, Boston.

[3] Jena Ajit K. and Popescu Adrian, Traffic engineering for Internet applications, SPIE proceedings series, Internet Performance and Control of Net work Systems II, Robert D. van der Mei; Frank Huebner-Szabo de Bucs; Eds., Vol. 4523, 2001, pp. 67-78.

[4] Philippe Owezarski, Nicolas Larrieu, Measurement Based Approach of Congestion Control for enforcing a robust QoS in the Internet, Report LAAS No. 04722, International Conference on Internet Surveillance and Protection (ICISP'06), Cap Esterel (France), 27-29 August 2006.

[6] Hurst H. E., Long-term storage capacity of reservoirs, Transactions of the American Society of Civil Engineers, 1951, vol.116, pp 770-808.

[7] Ton Dieker, Simulation of Fractional Brownian Motion, Masters Thesis, Department of Mathematical Sciences, University of Twente, The Netherlands, 2004.
[8] T. Higuchi, Approach to an irregular time series on the basis of the fractal theory, Physica D, 1988, vol.31, pp. 277-283.

[9] P. Abry, P. Flandrin, M. S. Taqqu and D. Veitch, Wavelets for the Analysis, Estimation, and Synthesis of Scaling Data, K. Park and W. Willinger (editors), Self-Similar Network Traffic and Performance Evaluation. John Wiley \& Sons, New York, 2000, pp. 39-88.

[10] P. Abry and D. Veitch, Wavelet Analysis of Long-Range Dependent traffic. IEEE Transactions on Info mation Theory, 1998, vol.44, No 1, pp.2-15.

[11] Stilian Stoev, Murad Taqqu, Cheolwoo Park and J.S. Marron, Strengths and Limitations of the Wavelet Spectrum Method in the Analysis of Internet Traffic, Technical Report \#2004-8, March 26, 2004.

[12] Kihong Park, Gitae Kim, Mark Crovella, On the relationship between file sizes, transport protocols, and self-similar network traffic, Fourth International Conference on Network Protocols (ICNP'96). 1996, pp. 171-180.

[13] Vern Paxson and Sally Floyd, Wide-Area Traffic: The Failure of Poisson Modeling, IEEE/ACM Transactions on Networking, June 1995, Vol. 3 No. 3, pp. 226-244.

[14] UNC Network Data Analysis Study Group, University of North Carolina, http://www- dirt.cs.unc.edu/net_lrd/ (visited on May 23, 2005).

[15] W. Willinger, M. S. Taqqu, W. E. Leland, and D. V. Wilson, Selfsimilarity in high speed packet traffic: Analysis and modeling of ethernet traffic measurements, Statistical Science, 1995, Vol. 10, pp. 67-85.

[16] Dario Rossi, Luca Muscariello, Marco Mellia, On the Properties of TCP Flow Arrival Process, IEEE International Conference on 
Communications (ICC 2004), Paris - France, June 20-24, 2004.

[17] Ashok Erramilli, Onuttom Narayan, and Walter Willinger Experimental Queueing Analysis with Long-range Dependent Packet Traffic, IEEE/ACM Transactions on Networking, April 1996, Vol. 4, No. 2, pp. 209-223.

[18] O. Rose, Estimation of the Hurst Parameter of Long-Range Dependent Time Series, Report No. 137, February 1996, Institute of Computer Science, University of Wurzburg.

[19] E. Willekens and J. Teugels, Asymptotic expansions for waiting time probabilities in an M/G/1 queue with longtailed service time, Queueing Systems 10, 1992, pp. 295-312.

[20] An Ge, Franco Callegati, and Lakshman S. Tamil, On Optical Burst Switching and Self-Similar Traffic, IEEE Communications Letters, March 2000, Vol. 4, No. 3, pp. 98-100.

[21] R.J. Gibbens, Traffic characterisation and effective bandwidths for broadband network traces, Stochastic Networks, Theory and Applications, 1996, pp. 169-179, Oxford Science P ub.

[22] Brocklebank J. and D. Dickey. SAS System for Forecasting Time Series. SAS Institute Inc. Cary NC. 1986.

[23] Jin Cao, William S. Cleveland, Dong Lin, and Don X. Sun, On the Nonstationarity of Internet Traffic, Proc. ACM SIGMETRICS `01, 102-112, 2001.

[24] Walter Willinger, Vern Paxson, and Murad Taqqu, Self-similarity and Heavy Tails: Structural Modeling of Network Traffic, Adler, R., Feldman, R., and Taqqu, M.S., (editors), In A Practical Guide to Heavy Tails: Statistical Techniques and Applications, Birkhauser, 1998.

[25] Cox D., Long-Range Dependence: a Review. H. A. David and H. T. David (eds.), In Statistics: An Appraisal, Iowa State Statistical Library, The Iowa State University Press, 1984, pp.55-74.

[26] Antoine Scherrer, Antoine Fraboulet, Tanguy Risset, Multi-phase On-chip Traffic Generation Environment, Laboratoire de l'Informatique du Parallélisme, École Normale Supérieure de Lyon, INRIA, Research Report No. 2006-22, June 2006.

[27] H. Kettani and J. A. Gubner, A Novel Approach to the Estimation of the Hurst Parameter in Self-Similar Traffic, Proceedings of the 27th Annual IEEE Conference on Local Computer Networks (LCN 2002), Tampa, Florida, November, 2002, pp.160-165. 